\title{
Begrüßung: Vom Wandel der modalen Zeiten Oder: Wie hoch das Wort zu schätzen sei
}

\section{Sprache verstehen - verstehen, was das meint}

Dass sich Linguisten mit der Sprache, ihrem systemhaften Aufbau und ihrer Strukturierung auf verschiedenen Ebenen beschäftigen, muss man eigentlich gar nicht erwähnen. Wer sollte das sonst tun?

Auch wenn man das feststellt, steckt dahinter schon etwas, was im Rahmen der 45. Jahrestagung des IDS im Jahr 2009 bei ihrem Thema „Sprache intermedial: Stimme und Schrift, Bild und Ton" allerdings noch eine größere Rolle spielt. Hier gilt nämlich besonders, dass die Erkennbarkeit bestimmter Bestandteile oder Aspekte sprachlicher Interaktion, die Möglichkeit, sie zur Beobachtung festzuhalten, zu wiederholen oder dem Experiment zugänglich zu machen, die Voraussetzung dafür ist, sie eigentlich erst mit einem wissenschaftlichen Blick zu erfassen. Was heißt das praktisch? Manches davon hat bei den Themen unserer Tagungen in den vergangenen Jahren ebenfalls eine Rolle gespielt. Gelegentlich ganz explizit, so wenn es beim „Text-Verstehen“ um „Grammatik - und darüber hinaus" " geht, häufiger implizit, etwa, wenn klar wird, dass sich mit der Existenz großer Sprachkorpora bestimmte Fragen übethaupt erst stellen lassen, wenn man die Chance auf eine empirisch begründete Antwort haben will, ${ }^{2}$ oder wenn man sieht, dass erst die Nutzung bildgebender Verfahren der Neurophysiologie Antworten z.B. auf die Frage der Interaktion zwischen Prosodie und Grammatik erlaubt. ${ }^{3}$ Man sieht auch hier schon, dass es nicht zuletzt neue technische Möglichkeiten der Dokumentation und Analyse sind, die den wissenschaftlichen Prozess in ungeahnter Weise voranbringen. Dass die Materialität der Kommunikation ein wesentlicher Faktor sprachlicher Entwicklung ist, ist ein einigermaßen gängiger Gedanke; ein doch deutlicher Schub in den technischen Dokumentationsmöglichkeiten hat uns zumindest praktisch auch alle gelehrt, dass die Möglichkeiten der Erfassung Antworten auf Fragen zu geben erlauben, die man ohne sie kaum in das ernsthafte Spektrum der eigenen wissenschaftlichen Tätigkeit aufgenommen hätte.

Was die Erhebungs-, Dokumentations- und Analysemöglichkeiten angeht, so gilt das in ganz besonderem Maße für die wissenschaftliche Bearbeitung gesprochener Sprache. Harald Thun (1993) hat in erhellender Weise dargelegt und nachvollzogen, wie die Miniaturisierung der Tonaufnahmegeräte

Siehe Blühdorn/Breindl/Waßner $(\mathrm{Hg}$.) (2006).

Siehe Kallmeyer/Zifonun (Hg.) (2007).

Siehe Kämper/Eichinger (Hg.) (2008). 
die Forschungspraxis in der Dialektologie verändert hat. Aber daran sieht man auch schon, dass es nicht die Techniken allein sind, sondern die Techniken in Interaktion mit den methodologischen und linguistischen Diskussionsständen. ${ }^{4}$ Und so haben wir seit einiger Zeit jedenfalls eine genauere Vorstellung zur Distribution und Schichtung regionaler Sprachformen. ${ }^{5}$

Es ist das eine sachlich gut passende Stelle um anzumerken und meiner Freude darüber Ausdruck zu geben, dass auf der Jahrestagung 2009 des IDS der Hugo-Moser-Preis an Alexandra Lenz verliehen werden konnte, eine junge Sprachwissenschaftlerin, deren Arbeiten in diesem Bereich deutliche Zeichen gesetzt haben. Den Preis bekam sie für ein Vorhaben zugesprochen, in dem ein zentraler und für verschiedene grundlegende Funktionen zur Verfügung stehender Bereich des verbalen Wortschatzes des Deutschen untersucht werden soll, nämlich der der Besitzwechsel-Verben.

Die Darstellungsweise meiner bisherigen Ausführungen, die den Einbezug weiterer Falktoren in die sprachwissenschaftliche Analyse als eine Art Ausgreifen von einem linguistischen Kern aus beschrieben hat, ist aber nicht hinreichend, wenn man erläutern will, welche Idee die diesjährige Tagung leitet. Zwar stimmt auch für diesen Kontext, dass der ganz andere Blick ohne die problemlose Verfügbarkeit, Analysierbarkeit und Reproduzierbarkeit von Video-Daten kaum denkbar ist, aber sich nur darauf zu berufen, wäre eine zu technizistische Sicht einer konzeptuellen Wendung. Beim Verstehen der Interaktionen in unserer Welt ist die Sprache ein - wenn auch phänotypisch und strukturell - herausgehobener Modus im Inventar der verständnissichernden Techniken. Wir verstehen, wenn man so will, was geschieht und dabei werden wir auch, aber nicht nur, sprachlich instruiert. ${ }^{6}$

\section{Verstehen-Wollen und Verstanden-werden-Wollen}

\subsection{Die Schrift und die Reduktion der Modi}

Fokussiert wird auf der diesjährigen Tagung aber eben jene von der Untersuchung der Sprache und ihres variierenden Gebrauchs aus gesehen andere Seite, nämlich die, dass man sich beim Verstehen- und Verstanden-WerdenWollen all der verschiedenen Möglichkeiten bedient, die einem zur Verfügung stehen - und eigentlich tut man das im Normalfall, ohne sich dabei all die Optionen bewusst zu machen, die man nutzt.

4 Im Hinblick auf die Verfügbarkeit von Korpora vgl. dazu Eichinger (2007).

5 Das IDS leistet dazu einen Beitrag mit dem Projekt „Deutsch heute“, das die Basis für eine präzisere Beschreibung standardsprachlicher und -naher Sprechformen bietet. Vgl. Eichinger/Kallmeyer (Hg.) (2005).

$6 \quad$ Wie sich das im Ablauf der Tagung entfaltet, wird in der Einführung der Herausgeber erläutert. 
Bei der Interaktion mittels der Schrift, also beim Lesen von Büchern zum Beispiel und beim Schreiben fällt das nicht so sehr auf. Man kann allerdings beim Lesenden und wohl auch beim Schreibenden manchmal ein Stirnrunzeln oder auch ein Lächeln entdecken, das erkennbar einen Teil der über die Schrift vermittelten Verständigung darstellt. Und es mag durchaus schriftliche Texte geben, die mit solchen Reaktionen rechnen und auf ihre Möglichkeit Bezug nehmen. Beim Alleinlesen, das seit dem beginnenden neunzehnten Jahthundert unseren Umgang mit Geschriebenem und Gedrucktem prägt, wird man nicht viel mehr von dem finden, was man multimodal nennen könnte. Das gilt zumindest, wenn man darunter die mimischen und gestischen Aktionen und Reaktionen der Beteiligten versteht. Gedruckte Texte jedenfalls sind ja im Prinzip von ihrem Autor und seinen auf der angedeuteten Linie liegenden Möglichkeiten abgeschnitten.

Schriftlichkeit selbst ist prototypisch vom Modus der Distanz geprägt und hat daher in entfalteten Schriftkulturen wie der Deutschen auch Techniken entwickelt, die das deutlich signalisieren. Die Tiefe der Orthographien, in denen die Sprachen der europäischen Schriftkulturen kodiert werden, ist ein Mittel zu dieser Auszeichnung.

\subsection{Die anderen Seiten schriftlicher Interaktion}

\subsubsection{Typo- und Orthographisches}

Allerdings hat der Autor, auch über Fragen der gewählten Sprachform und des praktizierten Stils hinaus, durchaus Möglichkeiten, über Eigenheiten der Materialität gedruckter Texte zumindest die Leseerwartung zu steuern. Eine nicht unwichtige Rolle spielt dabei die typografische Gestaltung. Man kann damit mancherlei signalisieren.

Um ein historisches Beispiel heranzuziehen, könnte man darauf verweisen, dass zu Zeiten, als gebrochene Schriften den Normalfall für die Schreibung des Deutschen darstellten, Wörter aus anderen Sprachen, nicht zuletzt aus dem Lateinischen, in lateinischer Schrift wiedergegeben wurden. Aber auch bis in unseren heutigen Alltag des Schreibens reicht diese Erscheinung hinein. Wir, die wir nun die meisten unserer Texte als Computerausdrucke, also irgendwie gedruckt sehen - die Schreibmaschine machte ihrem Namen noch Ehre, mit ihr schrieben wir noch -, haben in den letzten Jahrzehnten eine typographische Entwicklung mitgemacht, die uns auch signalisiert, dass es nun um „Gedrucktes" geht. So waren die Anfänge des schreibenden Computer-Alltags geprägt von zwei Arten von typographischen Einstiegen. Als Schreibmaschinenschreiber wurden wir abgeholt mit einer einfachen Serifenschrift vom Typ Courier, die uns unser Tun als eine Art avanciertes Schreibmaschineschreiben erscheinen ließ. Die - kärgliche Modernität des Neuen repräsentierte gleichzeitig eine dünne und eckige serifenlose Schrift, wobei beide Fonts ihre Beliebtheit wohl ihrer Affinität 
zu den Möglichkeiten des Nadeldruckers verdankten. Zwischenzeitlich sind wir nun in die Druckwelt hineingewachsen, indem wir eine Buch-Serifenschrift wie Times New Roman als unsere normale Schreibschrift nutzen und eine gestaltete, wenn auch etwas gröbere - serifenlose Schrift vom Typ Arial als die moderne Variante, die zudem - wenn man die üblichen Titelund Überschriften-Vorlagen in gängigen Schreibprogrammen betrachtet - gleichzeitig die Funktion solcher Schriften als gedruckte Hervorhebungschriften teilen.

Aber die Nutzung der materialen Eigenheiten graphischer Kommunikation geht deutlich darüber hinaus. Ein ebenso einschlägiges wie neues Beispiel dafür stellt die Handhabung der Orthographie dar: so haben die größeren gedruckten Massenmedien immer schon eine Hausorthographie mit bestimmten Eigenheiten gehabt, und jetzt im Gefolge der Diskussion um die Orthographiereform, hat die Wahl einer nicht den aktuellen Regelungen folgenden orthographischen Praxis deutlich an Informationsgehalt gewonnen.

Bei Büchern zum Beispiel spielt dabei auch die weitere materiale Kontextualisierung eine Rolle - vom gesetzten gebundenen Buch bis zur im einfachen Schreibprogramm formatierten und gehefteten Broschüre reicht hier die Bandbreite, die unsere Rezipientenhaltung nicht zuletzt im Hinblick auf die Ernsthaftigkeit der übermittelten Information steuert.

\subsubsection{Interaktion der Zeichentypen}

Mehr noch wird diese Art der Rahmung konstitutiver Teil des modalen Ensembles, wenn wir in den Raum der öffentlichen Schriftlichkeit kommen, der sich mit öffentlichen Anschriften, Hinweisschildern und dergleichen beschäftigt. In den Forschungen zum lingustic landscaping hat sich dazu ja direkt eine eigene Forschungsrichtung entwickelt. Wir wollen dem hier nicht weiter nachgehen, man kann aber darauf hinweisen, dass mit der Integration ikonischer Elemente und anderer analoger Mittel im Prinzip eine Ebene genutzt wird, die im Hinblick auf traditionellere Schriftlichkeit als eine Möglichkeit der Differenzierung von Texttypen beschrieben wird. In diesem Kontext werden dabei aber eher Fragen gestellt, die darauf zielen, welche Texttypen etwa durch die Nutzung von Tabellen, Graphiken, Illustrationen oder dergleichen gekennzeichnet seien.

Da wir den Weg gewählt haben, uns von traditionelleren Aspekten der Sprachwissenschaft her dem diesjährigen Thema anzunähern, sind die Bemerkungen, die wir gemacht haben, nicht aus dem die Tagungskonzeption eigentlich leitenden Konzept der Multimodalität her geschrieben, sondern entlang traditioneller Argumentationsstränge, die zwar über lange Zeit hin bedeutsam waren, nun aber seit etwa zwei Jahrhunderten deutlich an gesellschaftlicher Geltung verloren haben. 
Die aufgeklärte Rhetorik - und daran angelehnte Interaktionstheorien wie etwa die des Dramas - betrachten die sprachliche Vermittlung auch als einen von Regeln gesteuerten und getriebenen Umsetzungsmechanismus für Gefühle. Wenn eine klassische Formulierung in diesem Kontext lautet, si vis me flere, dolendum est primum itsi tibi, ist klar, dass hier eine harte sprechakttheoretische Kausalverbindung angenommen wird, die ihre Antwort in unmittelbarer Körperlichkeit findet. In der Situation des Lesenden, aber auch des Zusehenden ist das normalerweise aber auch das Ende der Interaktion. Dass auch die Schrift selbst in einen neben der zentralen Kodierungsfunktion für Inhalte stehenden Modus eintreten kann, konnten wir schon an den Beispielen zur Nutzung ikonischer Elemente des Schreibens und des Druckens sehen. So kann man, um das noch in eine etwas andere Richtung weiter zu führen, handschriftlich niedergelegte Nachrichten nicht nur in einem graphologischen Sinne im Hinblick auf den Schreiber deuten, ein fest aufgedrückter Bleistift, hervorgehobene Majuskeln oder Unterstreichungen und die Wahl von Satzzeichenhäufungen können durchaus im Hinblick auf die Ausdrucksfunktion gelesen werden, die etwa mit einem appellativen Text verbunden ist. Nicht umsonst nutzt die gedruckte Werbung die Variation solcher Mittel in entsprechender Weise. Von diesem eher indexikalischen Modus der Interaktion ist kein besonders weiter Weg zu dem analogischen Konzept der Verständigung über ikonische Zeichen, von den Herzen in Liebesbriefen über die Emoticons und Piktogramme der Moderne, aber auch zu den Bildgedichten durch die Zeiten. ${ }^{7}$

Und wenn uns auch in der Moderne das Wissen um und der Glaube an die Lesbarkeit der Welt, von der die emblematischen Zeiten unserer Kultur lebten, abhandengekommen sind, wir haben nicht nur ein Inventar an Symbolen lesen gelernt und nutzen sie je nach unseren intertextuellen Erfahrungen, vielmehr lösen wir auch Bilder und Bildfolgen nach unseren Vorstellungen von Szenen und Ablaufstahmungen auf bzw. interpretieren sie je nach Kontext auf diesem Hintergrund. So haben wir etwa auch mit Gesetzen filmischer Ikonographie leben gelernt und können ihre Muster nutzen. Aber das ist eigentlich wieder ein Umschwung in der Ordnung der sinnlichen Wahrnehmung, der gelernt werde musste: und so ist es nicht überraschend, dass aus den Anfangstagen öffentlicher Filmvorführung davon berichtet wird, die Zuschauer seien in Angst vor einem auf sie zufahrenden Zug geflohen. Welchen Weg wir seither gegangen sind, mag man daran sehen, dass wir unlängst staunend vor der Meldung standen, dass sich die Avatare zweier auch in unserer ersten Welt verheirateter Personen scheiden lassen wollten, wobei offenbar den beiden Beteiligten nicht ganz klar war, was das für ihre „unavatare“ Wirklichkeit zu bedeuten hätte.

7 Das wäre die Stelle, wo man über die Mittel von ideographischen und Alphabetschriften räsonieren könnte - aber das würde zu weit führen. 


\section{Die Reihenfolge der Wahtnehmung}

\subsection{Die Welt im Buch}

Wir können das auf sich beruhen lassen. Wir waren ja bei jener historischen Stufe angekommen, zu der die Lesbarkeit des - emblematischen - Bildes die alltägliche Welt verließ und sich als symbolische Rekonstruktion in esoterischere Räume eingeweihter Deuter zurückzog. Was allerdings bleibt, ist die Vorrangstellung des Auges als des Sinnesorgans, mittels dessen wir uns Wissen einverleiben, und nicht das Ohr, dessen Wahrnehmung insgesamt als vorübergehend und nicht rückholbar angesehen wird. Das Auge allerdings, es nimmt mit der Vorherrschaft der gelesenen (und geschriebenen) Sprache die Welt geradezu besser durch die sinnvollen Buchstabenfolgen als durch einen direkten Blick auf die Welt wahr. Und so signalisiert es ganz deutlich eine nicht nur logozentrische, sondern auch "skriptozentrische“ Sichtweise, wenn Carl Philipp Moritz im Jahr 1783 in sein "Neues A.B.C. Buch" folgendermaßen beginnen lässt:

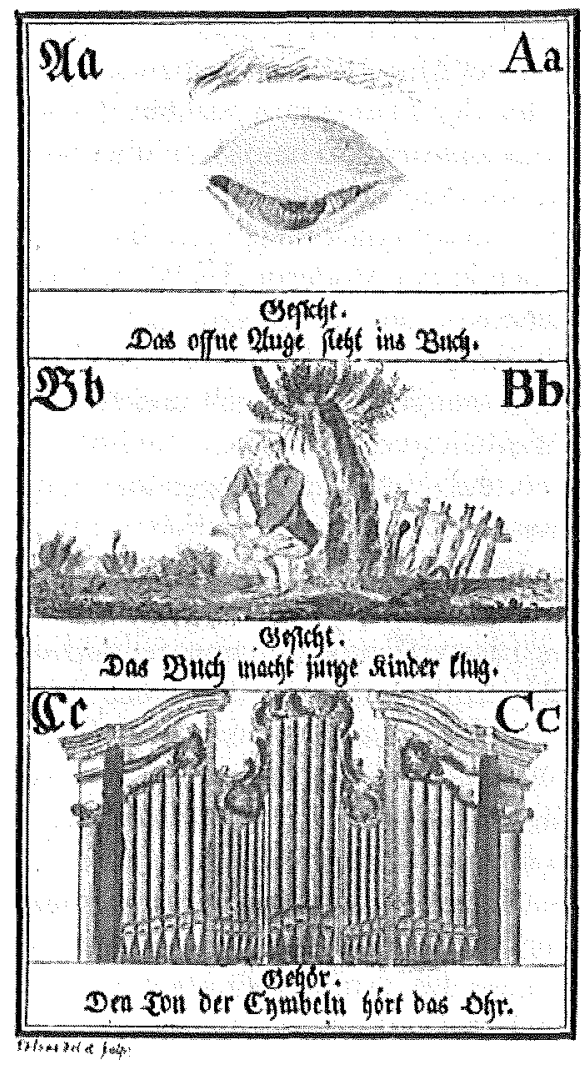


Erstes Bild.

In diesem Buche stehen Bilder und Buchstaben. Das erste Bild stellt das Auge vor, womit ich die Bilder sehe.

\section{Zweites Bild.}

Das zweite Bild stellt einen Knaben vor, der unter einem Baum sitzt, und in einem Buche lieset. Der Knabe hält den rechten Zeigefinger auf das Buch, damit er in der rechten Zeile bleibe. Der Knabe ist sehr aufmerksam und gaft nicht umher. Bei den Bildern stehen Buchstaben. Unter den Bildern stehen Worte. Wer nicht lesen kann, der besiehet nur die Bilder. Wer aber lesen kann, der lieset auch die Worte, die darunter stehen. Das offene Auge sieht ins Buch. Mein Auge ist offen, und ich sehe damit ins Buch. Das Buch macht junge Kinder klug. Ich will in diesem kleinen Buche fleißig lesen lernen, damit ich noch mehr Bücher lesen kann, wodurch ich klüger werde.

(www.hs-augsburg.de/ harsch/germanica/Chronologie/18]h/Moritz/mor_ab0a.html\#01)

Das Buch ist es, dessen Wahrnehmung über das Auge die ,jungen Kinder"“ klug macht. Das ist, wie marginal das Beispiel als solches sein mag, durchaus als Symptom lesbar, dass wir in ein Zeitalter der Schriftlichkeit eintreten, die auch durch eine weitgehende Alphabetisierung der Bevölkerung getragen wird. So ist es nicht zuletzt zunächst die Orthographie, die geregelt wird, es geht um das Lesen und das Schreiben.

\subsection{Die Dichte der Information}

Man kann dann im Verlauf des neunzehnten Jahrhunderts sehen, wie sich die Untersuchung, ,wirklich“ gesprochener Sprache, wie sie zu dieser Zeit die Dialektologie unternimmt, müht, der Andersartigkeit des Phänomens mit schriftlichen Mitteln beizukommen, was bei der Aufnahme zweifellos ein noch größeres Problem ist als bei der wissenschaftlichen „Weiterverarbeitung". Erst allmählich haben sich die Aufnahmebedingungen für gesprochene Sprache soweit perfektioniert, dass sich allmählich das Problem auf die andere Seite verschoben hat. Die Menge und die Informationshaltigkeit des Aufgenommenen erschwert uns nun die klassifizierende Beschreibung - und fordert auf jeden Fall einen außerordentlich erhöhten Analyseaufwand, der sich zumindest derzeit noch nicht über automatische Prozeduren in hinreichendem Maße reduzieren lässt. Dieses Problem verschärft sich noch einmal dramatisch, wenn die Möglichkeit der Video-Aufnahme eine multiaspektuelle Kontextualisierung verlangt. So wird die Verbindung einer möglichst weitgehenden Interaktionsanalyse mit der Typisierung der Ergebnisse sicher nicht leichter. Gerade ihr sollten wir aber nachgehen wollen, wenn wir verstehen wollen, wie wir in solchen medial überlagerten Kontexten funktionieren können, wenn wir sehen wollen, wie wir unter diesen Umständen verstehen können, worum es geht. Natürlich erkennen 
wir auch in dieser Interaktion Muster, so haben wir etwa gut gelernt, bestimmte filmische Abläufe auch in ihrer spezifischen Räumlichkeit und körperlichen Interaktion zu erkennen. Andererseits ist Schriftlichkeit in ihrer öffentlichen Funktion zum Teil sehr stark an ikonische oder indexikalische Kontexte rückgebunden, so dass wir mit geringerer Explizitheit zurechtkommen können.

\subsection{Wandel der Alltäglichkeit}

Da die Behertschung von Schriftlichkeit seit dem Ende des achtzehnten Jahrhunderts die Teilhabe an der bürgerlich-demokratischen Entwicklung der folgenden Zeit ermöglichte, markiert ihre Dominanz einen wichtigen diskurshistorischen Schritt: Teilhabe am kommunikativen Gedächtnis und der Niederlegung des kulturellen Gedächtnisses in diesem digitalen Aufschreibsystem. Mit der Zunahme des Hörens und Bildersehens und nicht zuletzt auch des medial vermittelten Sprechens in den medialen Schritten des zwanzigsten Jahrhunderts, werden die Verhältnisse von individueller sowie näheorientierter und fest gewordener distanzorientierter Formen der Interaktion neu gemischt. Unter diesem Aspelst ist die technische Meisterung der Beobachtung der verschiedensten Modalitäten bei (auch) sprachlicher Interaktion zwar ein wichtiger Punkt für den Aufschwung dieses Tuns, aber andererseits ihrerseits auch nur ein Aspekt der Entwicklung, der dieser Art von Beobachtung eine Bedeutung verleiht, die darüber hinausgeht, sie als paralinguistisch zu sehen. Neben manch anderem mögen die in diesem Band versammelten Beiträge unserer Jahrestagung auch als Anregungen zu einer diskurs- und techniksensiblen theoretischen Modellierung dieser Sachverhalte zu lesen sein. ${ }^{8}$

\section{Schluss}

Zum Zeitpunkt der Dokumentation dieser Tagung kann man sagen, dass die Vielfalt, die sie gekennzeichnet hat, für Sprachwissenschaftler der verschiedensten Provenienz nicht nur Anregung, sondern auch Wissenswertes zu bieten hatte. $\mathrm{Zu}$ danken ist allen, die auf dieser Tagung referiert haben, nicht zuletzt, den Nichtlinguisten darunter, die uns halfen, einen fachspezifischen Tunnelblick zu vermeiden. Wie immer haben die für die Organisation der Tagung verantwortlichen Mitarbeiterinnen und Mitarbeiten des Hauses für einen glatten und problemlosen Ablauf gesorgt, wofür Ihnen Dank gebührt. Die inhaltliche Planung und Vorbereitung lag in den Händen von Arnulf Deppermann und Angelika Linke. Für die konzeptionelle

8 Ihr Zusammenhang und der Aufbau der Tagung wird in dem Beitrag von Arnulf Deppermann und Angelika Linke dargestellt. 
und organisatorische Planung und Leitung des Ablaufs sei ihnen ebenfalls herzlich gedankt. Zu guter letzt möchte ich dankbar daran erinnern, dass die Stadt Mannheim und ihr Oberbürgermeister nicht nur nach guter Gewohnheit die Teilnehmer zu einem Empfang geladen hat, sondern dem Institut geholfen hat, die noch immer etwas ungewohnte Bürde der mit dem Tagungsort Rosengarten verbundenen finanziellen Lasten zu tragen. All die Genannten haben dazu beigetragen, dass die Tagung erfolgreich und in guter Atmosphäre stattfinden konnte.

\section{Literatur}

Auer, Peter (1993): Über $\cap$. In: Schlieben-Lange (Hg.), S. 104-138.

Blühdorn, Hardarik/Breindl, Eva/Waßner, Ulrich (Hg.) (2006): Text - Verstehen. Grammatik und darüber hinaus. (= Jahrbuch des Instituts für Deutsche Sprache 2005). Berlin/New York.

Bühler, Karl (1934): Sprachtheorie. Die Darstellungsfunktion der Sprache. Jena.

Deppermann, Arnulf/Linke, Angelika (i.d.Bd.): Einleitung: Warum „Sprache intermedial"?

Eco, Umberto (1994): Die Suche nach der vollkommenen Sprache. München.

Eco, Umberto (2000): Kant und das Schnabeltier. München.

Friederici, Angela D. (2008): Sprache und Gehirn. In: Kämper/Eichinger (Hg.), S. 51-72.

Eichinger, Ludwig M. (1993): Vor Augen gefühtt bekommen. Text-Bild-Kombinationen als Merkhilfen. In: Dirscherl, Klaus (Hg.): Bild und Text im Dialog. (= PINK 3). Passau, S. 429-449.

Eichinger, Ludwig M. (2007): Linguisten brauchen Korpora und Korpora Linguisten Wege zu wohl dokumentierten und verlässlichen Aussagen über Sprache. In: Kallmeyer/Zifonun (Hg.), S. 1-8.

Eichinger, Ludwig M./Kallmeyer, Werner (Hg) (2005): Standardvariation. Wie viel Variation verträgt die deutsche Sprache? (= Jahrbuch des Instituts für Deutsche Sprache 2004). Berlin/New York.

Gessinger, Joachim (1994): Auge \& Ohr: Studien zur Erforschung der Sprache am Menschen 1700-1850. Berlin/New York.

Harras, Gisela (2004): Handlungssprache und Sprechhandlung. Eine Einführung in die theoretischen Grundlagen. 2. erw. u. durchges. Aufl. Berlin/New York.

Kallmeyer, Werner/Zifonun, Gisela (Hg.) (2007): Sprachkorpora - Datenmengen und Erkenntnisfortschritt. (= Jahrbuch des Instituts für Deutsche Sprache 2006). Berlin/New York.

Kämper, Heidrun/Eichinger, Ludwig M. (Hg.) (2008): Sprache - Kognition - Kultur. Sprache zwwischen mentaler Struktur und kultureller Prägung. (= Jahrbuch des Instituts für Deutsche Sprache 2007). Berlin/New York. 
Konopka, Marek/Strecker, Bruno (Hg.) (2009): Deutsche Grammatik - Regeln, Normen, Sprachgebrauch. (= Jahrbuch des Instituts für Deutsche Sprache 2008). Berlin/New York.

Lessing, Gotthold Ephraim/Mendelssohn, Moses/Nicolai, Friedrich ([1756/57] 1972): Briefwechsel über das Trauerspiel. Hrsg. u. komm. v. Jochen Schulte-Sasse. München.

Moritz, Karl Philipp (1794): Neues A.B.C. Buch, welches zugleich eine Anleitung zum Denken für Kinder enthält. 2. Aufl. Berlin. (Hier nach: www.hs-augsburg.de/ harsch/ germanica/Chronologie/18Jh/Moritz/mor_ab0a.html\#01, Stand: November 2009).

Schlieben-Lange, Brigitte (Hg.) (1993): Materiale Bedingungen der Sprachwissenschaft. (= Zeitschrift für Literaturwissenschaft und Linguistik 23, 90/91). Göttingen.

Schön, Erich (1993): Der Verlust der Sinnlichkeit oder: Die Verwandlungen des Lesers. Stuttgart.

Stetter, Christian (1997): Schtift und Sprache. Frankfurt a.M.

Thun, Harald (1993): Was hat sich in der Dialektologie durch die Benutzung von Tongeräten geändert? In: Schlieben-Lange (Hg.), S. 139-156.

Weinrich, Harald (1997): Lethe. Kunst und Kritik des Vergessens. München.

Widmaier, Rita ( $\mathrm{Hg}$ ) (1990): Leibniz korrespondiert mit China: der Briefwechsel mit den Jesuitenmissionaren (1689-1714). Frankfurt a.M. 